\title{
A semi-automatic 2D/3D annotation framework for the geometric analysis of heritage artefacts
}

\author{
Adeline Manuel \\ Team GAMSAU \\ UMR MAP MC/CNRS 3495 \\ Marseille, FRANCE \\ adeline.manuel@map.cnrs.fr \\ Livio De Luca \\ Team GAMSAU \\ UMR MAP MC/CNRS 3495 \\ Marseille, FRANCE \\ livio.deluca@map.cnrs.fr
}

\author{
Anas Alaoui M'Darhri \\ Team GAMSAU \\ UMR MAP MC/CNRS 3495 \\ Marseille, FRANCE \\ anas.alaoui@map.cnrs.fr
}

\author{
Violette Abergel \\ Teams GAMSAU / ARIA \\ UMR MAP MC/CNRS 3495 \\ Lyon, FRANCE \\ violette.abergel@1yon.archi.fr
}

\author{
Fabien Rozar \\ UMR MAP MC/CNRS 3495 \\ Montpellier, FRANCE \\ fabien.rozar@gmail.com
}

\begin{abstract}
Documentation and monitoring of heritage objects involve many actors on multidisciplinary aspects. The progress made over the years in the field of digital technologies has enabled many tools for analysis, management and dissemination of information gathered around an object. These tools must allow users to semantically describe the object while allowing them to grasp its morphological complexity and the heterogeneity of the available analysis supports. This article introduces an approach for the semantic annotation of heritage objects by using the bijective relationship that can be established between a 3D representation of an object and the set of oriented images towards it, while maintaining a continuum of information between all phases of observation and description, from acquisition to visualization of semantically enriched representations. The main idea is to offer a versatile environment to help extraction of relevant information from images using geometric descriptors and semi-automatic point cloud processing methods.
\end{abstract}

Index Terms- $\mathrm{CH}$ documentation, semantic annotation, geometrical and visual descriptors, photogrammetry, segmentation

\section{INTRODUCTION}

Heritage asset monitoring and management are multidisciplinary fields by nature. They involve a plurality of actors, methods, and tools related to a wide variety of scientific issues and objectives. This context naturally leads to a phenomenon of dispersion of data, information and knowledge, which poses major problems in terms of sustainability. The heterogeneity of this context also causes difficulties in linking different levels of observation and analysis of the built environment by the various actors involved. Finally, the sensitive nature of the studied sites and their fragility, as well as their evolution over time, imply the need for regular monitoring that requires a constant supply of information.

\section{Gathering of analysis strategies}

Based on these findings, our main objective is to merge all the different aspects of the patrimonial survey (geometry, visual appearance, and semantics) within an integrated documentary approach built on an informative continuity that merges the acquisition, analysis and interpretation phases in a collaborative framework. This approach raises three main problematics :

- Semantic: To bring concrete meaning to geometric data

- Collaborative : To allow the various actors of the heritage to better communicate their results

- Interoperability: To provide a common platform, independent of the device and the operating system, so that collaborators can exchange their analyses in a transparent way.

\section{StATE OF THE ART}

Heritage assets study involves the collection of numerous data in addition to visual representations. This data must be structured, based on theoretical work on the naming of concepts and their interconnection [1]. The general goal is to associate semantic data with documents, which can take the form of simple vocabulary terms, or through ontologies, structuring terms and concepts related to a specific field of knowledge ( [2] [3] [4] ). Several studies have been conducted on this association, and more particularly on the semantic enrichment of 3D digitization ( [5] [6] [7] [8] ). [9] also offers the ability to integrate and annotate different types of 2D media in addition to the digital model.

In terms of web visualization, the new web technologies like the WebGL API have enabled the development of many viewers dedicated to the manipulation of 3D data ( [10] [11] [12] ), possibly annotated. Nevertheless, most of them consider $2 \mathrm{D}$ and $3 \mathrm{D}$ visualization as two distinct 
subjects. Let us however note [13], whose objective is to allow users to geo-reference archive photographs, then visualize them by transparency on a 3D representation of the globe, or [14] and [15] which also make it possible to visualize $3 \mathrm{D}$ and oriented images within a single scene.

However, if these viewers allow to spatialize 2D resources, they do not offer specific interaction modalities or annotation visualization. Finally, [16] offers all of these features, but uses meshes instead of raw data, on which annotations are overlaid as textures [17]. This data structuring does not allow a direct link to be established between the real object and its digital model and is therefore not sufficient to construct the expected digital epidermis around the studied object.

\section{MAIN APPROACH}

Our approach - the Aioli platform [18], currently under development - is based on the observation that the element most likely to federate all the above-mentionned actors is the "physical" entity of the heritage object under study. The ambition of this platform is to establish a link between the real object and its "digital ecosystem", in order to guarantee a continuum of information ranging from data acquisition to the construction of semantically enriched 3D digital representations.

This information continuum is established, from photographs of the studied object, thanks to a processing pipeline integrated into the Aioli platform. The latter responds to contextual issues by considering the study of an object (artifact or heritage building) as a "project", and by providing users with a working environment - a workspace - integrating all the above approaches.

\section{A. Workspace Preparation}

The first step is based on photogrammetry, which allows us, from an initial set of images, to construct a point cloud in three dimensions, a representation of the object acting as a digital clone [19]. The second phase of this implementation is the point cloud indexing, in order to establish a correspondence between the points of the cloud and the pixels of the images [20]. The bijective relationship thus established allows us to know, for each 3D point, the list of images on which the point appears, and for each of them, the corresponding pixels. Conversely, starting from one or more point(s) in an image, we can get a list of the corresponding 3D points (Figure 1).

\section{B. Spatializing semantic data}

This principle is more specifically used to allow users to draw annotations (observation areas) on an image and automatically reproduce them not only on the point cloud but also on all other images on which this area is visible. By allowing the various actors to associate to these annotations semantic descriptions coherent with their field of study, we finally spatialize information, by storing it as close as possible to the real object.

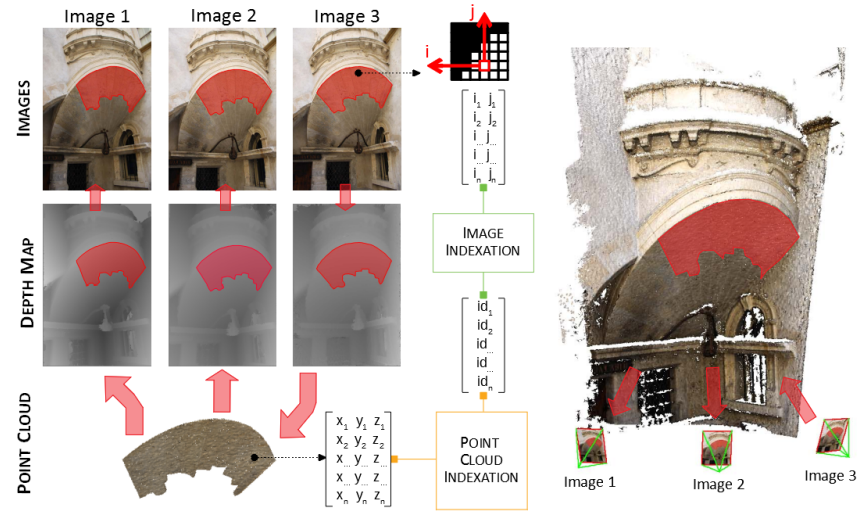

Fig. 1. Bijective relationship between images and point cloud

\section{Annotation Containers}

Once the working environment is ready, the users only have to define "annotation layers", named data structures. These structures allow to group in a semantic way the annotations which would have common aspects.

These layers give us a way to gather similar regions, and offer them to the users' browsers for display, with a familliar way of storing data, since the layers mechanism is already implemented in a lot of different softwares. They also are a good way to organize multi-user works, as they're holding different rights (read-only, read/write) for each user. In this way, the project owner can control more precisely who can see his work, and who can edit and enhance his analysis. Finally, they also are a way to hold formal description sheets, which normalize the annotations for each layer, as described later.



Fig. 2. Example of annotated layers on the same image [2]

For example, for the monitoring of architectural heritage [2], the user can create 3 layers, one layer named "Degradations", supporting all the annotations describing degradations of the material (cracks, detachment of matter, biological colonization...), one named "Material", created to describe the materials used for such constructions, and finally one named "Architectural elements", which will hold all the regions describing classical architectural elements (walls, columns, vaults...) (Figure 2). The comparison of these different layers then permits to identify the materials most exposed to particular alterations, or to target - with more accuracy - the 
architectural elements to be restored.

\section{Formal Description sheets}

The user-defined descriptors add a way to store information within an annotation, allowing the layer owner to define flexibly their own data structure, by adding fields: text, date, URL, concept from a thesaurus, and even attached documents (images, pdfs, sound, video). In this way, the user makes sure that their working tool will be adapted to their observations and to the needs of their study. Thus, all the annotations of a layer inherit the parent structure, which ensures a certain semantic uniformity. In the "Degradations" layer of the example above, 3 description fields could be defined: The type of degradation, the date of the survey and different observations related to the degradations. When a user characterizes a degradation, he must then fill these fields, fixed for each annotation of the layer.

This structure allows the user to visualize, collect and organize data in a semantic way. These are mainly qualitative data, related to the users' fields of study; but what about quantitative data describing the object's geometry, taking advantage of the three-dimensional resources?

\section{Automatic data enhancement: Geometrical \& VISUAL SUPPLY}

The characterization of a point cloud involves extracting data representative of all the points or the geometry at each point of the cloud.

\section{A. Computed Quantitative Descriptors}

For each point cloud, whether it is the complete point cloud of the object, or the 3D representation of an annotation, a set of "basic" descriptors can be extracted to bring out information related to the region involved.

We extract numerous data such as the number of points composing the $3 \mathrm{D}$ region, the barycenter, information related to the bounding box (position, center, magnitude, volume), the average orientation (average normal) and the average color automatically for each point cloud representation. In addition, for normal vectors and point colors, a range of representative values for the region is extracted to nuance the information provided by the simple average.

Thus, to describe each annotation, the user has, on the one hand, semantic data associated by himself through the description sheets and on the other hand, numerous data automatically generated by the system. All this data can then be used to define queries, for research, classification, and comparaison purposes.

\section{B. Visual Geometrical Descriptors}

Images of the object in the visible spectrum do not always allow a good appreciation of the different characteristics of the object, such as shapes or surface appearance, and can therefore sometimes make it difficult to select a region.
Thus, to facilitate analysis and interpretation operations, we propose the generation of images highlighting the geometric information extracted automatically on the point cloud.

The estimation of the values of curvature, roughness and ambient occlusion [21] for each point of the cloud are made comprehensible on a point cloud by the use of a color gradient. Thanks to the $2 \mathrm{D} / 3 \mathrm{D}$ relationship, this information is reprojected in the view of each of the images and thus generate images color-coding this geometric information of the point cloud (Figure 3). These images can then be used as support for the selection in the same way as photographies.

This supposes therefore to set up a dynamic viewer allowing at the same time to display the point cloud, the source images, but also the reprojection of the various "textures" of the images (normal, curvature, roughness, ambient occlusion, etc...).

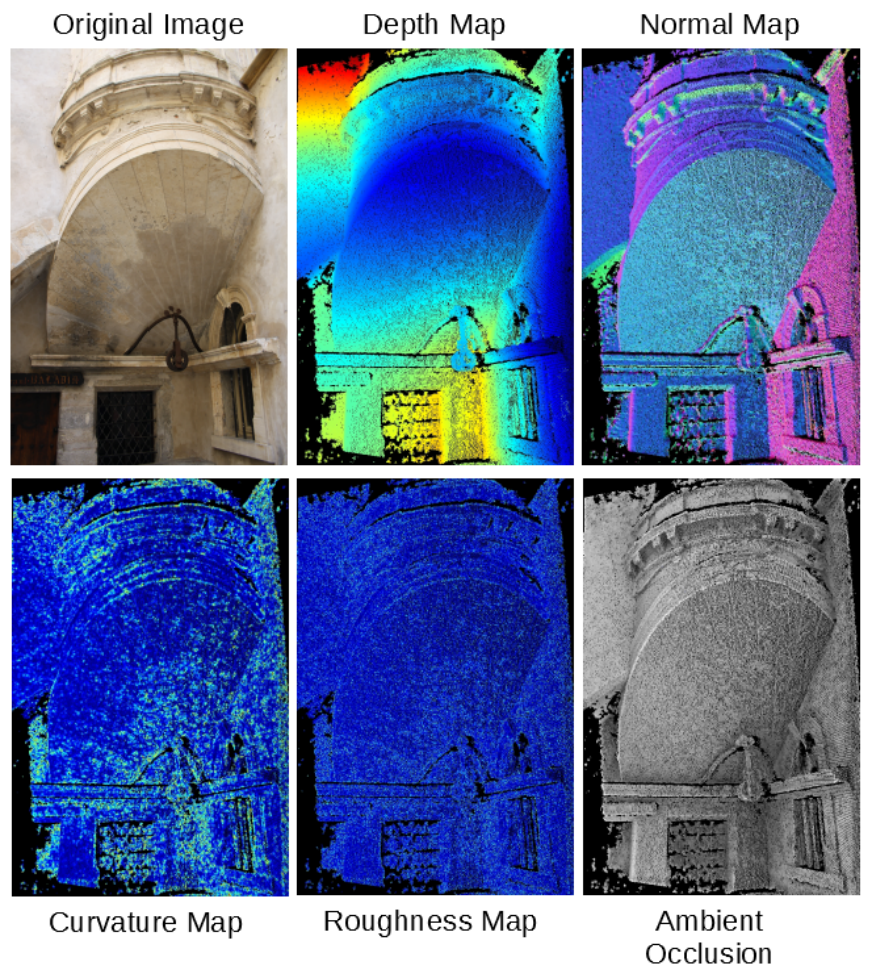

Fig. 3. Examples of reprojected geometrical descriptors

\section{Interaction with the information continuum}

The convergence of these analysis tools resulting from this new informational continuum implies the definition of a new approach concerning user interactions. More specifically, this led us to develop a hybrid 2D/3D viewer allowing users to take advantage of the bijective relationship by offering interaction tools that can be adapted to different types of device. 


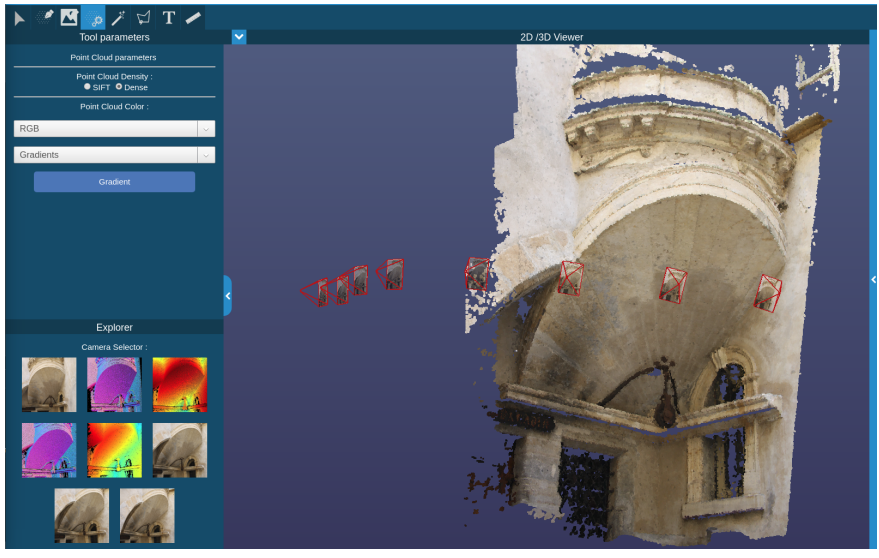

Fig. 4. User interface for the Aïoli platform

For this, the first phase was to literally translate within a dynamic viewer the relationship linking $2 \mathrm{D}$ and 3D, positioning and orienting the different iconographic resources relative to the point cloud, in order to create a coherent representative space (Figure 4). In this way, the user can position himself on each view and visualize in transparency the underlying 3D data. For this purpose, he benefits from all the available visual supports: both the original images and the images created by projection of the calculated geometric descriptors.

A second phase was to propose interaction tools adapted to the practice of annotation on 3D models. The 2D annotation tools were therefore completed by a real-time projection of the plot control points on the point cloud, in order to offer a 3D preview of the current annotation. As long as it is not validated, the trace remains editable. It is now also possible to define an annotation directly on the point cloud, and to edit it, before propagating it to all the project resources.

Finally, the multiplicity of profiles of actors that can intervene on the same project, and the complexity of some studied entities led us to propose more varied representation tools, allowing users to define their own graphic charter. Thus, users can now create annotation typologies, for which they can define the type of line (dotted or solid line) and thickness, spacing, color, opacity, filling, etc. Thus, during an annotation, the user selects in his personal list the relevant type of trace in order to obtain a coherence in the graphic representation. Such a space of visualization and interactions presents then a double interest: it allows not only to facilitate the study of a heritage object by facilitating the observation and the collaboration, but it also increases considerably the traceability and lasting quality, thanks to the safeguard of the various handling leading to the elaboration of a data. In fact, through this approach we aim to assign as much value to processes as to their results.

\section{Point Cloud Segmentation}

Segmentation methods offer automatic solutions for subdividing a digital model. The principle is based on the clustering of points in sets that share a common property (geometric or colorimetric). Many segmentation methods exist [22] and all face difficulties related to the precision of the point cloud generated (number of points, irregularity, noise, outlier points...).

In order to offer users a simple way to create annotations, the use of segmentations is currently being implemented on our platform. The idea is to automatically detect on the point cloud sets of points sharing a common characteristic and then provide the user with regions to annotate predefined by segmentation. Thus, for the time being, only a plane segmentation tool has been integrated in order to validate our approach.

\section{A. Plane segmentation}

A plane is the simplest surface to identify from a mathematical perspective in regards to its geometric characteristics. In order to overcome the difficulties aforementioned, our planesegmentation is based on an octree-based region growing method initially created in [23]. This method, described in [24] works in two iterative steps:

- Split : This step divides the input point cloud into sets of points in the form of an octree i.e. in the form of cubes containing points satisfying a same refinement criterion. In our case, this criterion is satisfied for a cube if the average distance of the points from their best fit plane is lower than the user-defined threshold. Starting initially from a cube containing all the points, each iteration subdivides the different cubes by testing, for each of them, the refinement criterion until all the cubes satisfy it. This process generally builds an irregular octree where each leaf contains the points closest to their best fit plane.

- Merge: This step groups the points identified as coplanar into regions. A region is built with the following process: it is initialized with an octree leaf and then iteratively constructed by exploring and adding neighbouring octree leaves as long as the merge criterion is met. In our case, this criterion is satisfied if the angle between the normal vector of the current region and the one of the considered octree leaf is lower than a user-defined threshold. The set of different regions is therefore obtained when all leaves of the octree have been tested and assigned.

\section{B. Integration with Aioli platform}

The result of this segmentation gives a clustering of the 3D points, forming different regions which can then be reproduced on each image thanks to the 2D/3D indexation mechanism (Figure 5). This feature allows automatic generation of an annotation layer grouping the regions detected by segmentation. The user only have to associate the semantic descriptions to each of the newly created layer regions. 


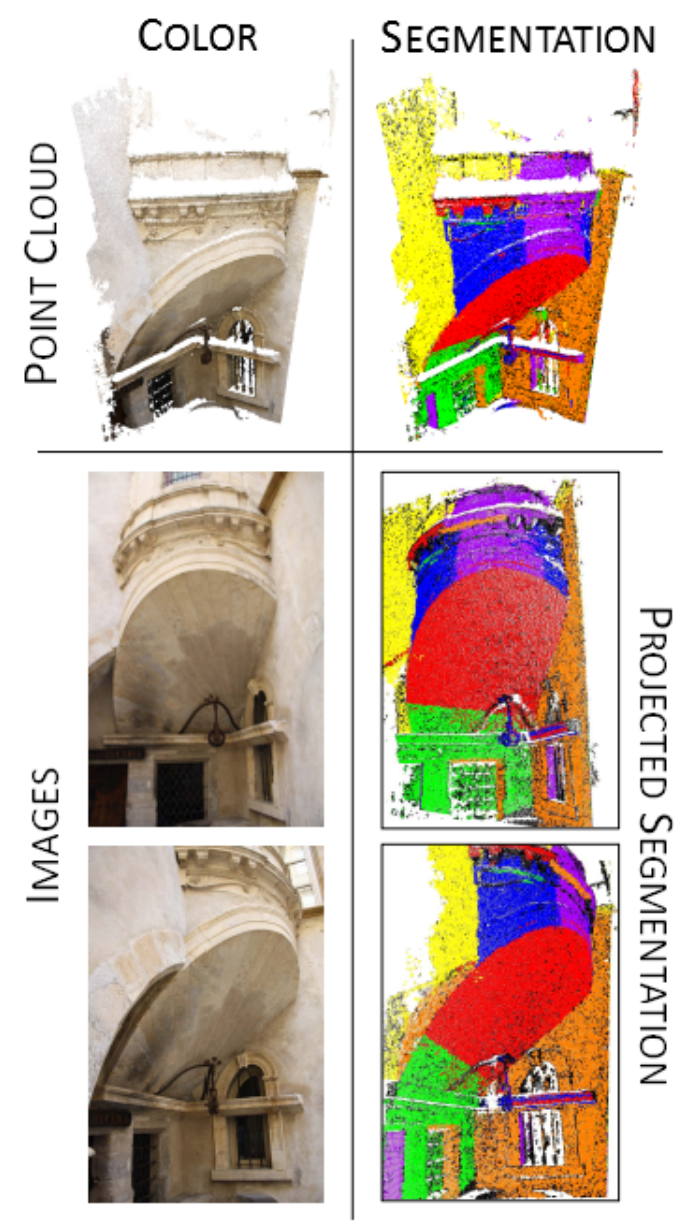

Fig. 5. Example of segmentation answer, and its reprojection on the images

The plane segmentation algorithm above-mentionned is one of many examples we could use. The Aioli system is built on Docker@ [25] to encapsulate the different software used to process the projects. We benefit from this flexible architecture by using all external dedicated software (photogrammetry engine MicMac, point cloud indexation, annotation propagation, and also segmentation plugins) as Docker images, so we can use all software components the same way. This allows us to not only run efficiently by preserving computing resources, but also be able to easily develop and import all kind of needed software, and more specifically segmentation algorithms as plugins processes for Aïoli.

Finally, if our toolset (geometric descriptors and segmentation) uses 3D data as a support for the information generation and the visualization of the results on the images, the inverse process can easily be envisaged thanks to the 2D/3D relationship. All processing performed on images can be easily projected onto the 3D model. Thus the image segmentation methods [26] could help in the 3D model segmentation and the machine learning methods generally applied to images rather than $3 \mathrm{~d}$ data [27] could allow to classify in a semi-automatic way the elements of the 3D model by detection in images (visible or geometric descriptors).

\section{SEMI-ASSISSTED SELECTION}

If one of Aïoli's specificities is to establish a link between semantic data and geometric data, it is essential to consider different annotation modalities adapted to these two fields: user-centered, when the annotation depends entirely on the user's field of expertise, automatic or assisted, when the annotation results from calculations.

Thus, the existing semantic annotation tools are essentially manual since they aim to bring semantically qualified information to the 2D and 3D data of the project. For now, three tools are available on the platform: point selection, polygon drawing, or free form. These three modalities are mainly carried out on photographs.

The next part introduces new tools for assisted annotation, based on the exploitation of segmentations and the implementation of graphical selection tools.

\section{A. From multisegmentation to annotation layers}

Today, the work performed on the geometric analysis of project data allows us to consider extending these methods with an assisted annotation tool. The latter will be based on the implementation of multi-segmentation plugins, like the one described above for plane detection.

The principle is simple: a preliminary segmentation operated on a point cloud can automatically suggest annotations to the user in order to select regions with similar geometric characteristics. If the annotation proposal is not sufficient for the user, he can modify it in order to fit his objectives, thanks to existing graphic tools. Once the regions have been selected, they can be enriched via their layer description sheets.

\section{B. Graphical selection}

Aïoli's main annotation functionality is based on the observation of objects by experts. Starting from this principle, the most suitable media to collect annotations are the images (photos) of the object. These annotations, made using the graphic tools mentioned earlier, are not automatic. However, it is possible to rely on geometric descriptors in order to make precise annotations according to particular features.

Some automatic selection tools available on large image editing software (Photoshop and others) would facilitate the detection and annotation of features on the Aioli platform. Among these tools we are mainly interested in magnetic lassos and magic wands, allowing, starting from a simple parameter (threshold), to select in a more or less precise and effective way regions of interest, in particular those which are not visible on the photographs, but stand out on the geometric descriptors reproduced.

A magic wand tool would not only allow to locate areas with close colors on the photos, but also to locate areas with close features on different textures. For example, this tool automatically selects a plane by applying it to the normal 
map, with the correct threshold parameter (Figure 6).

But we can go further, by complexifying a bit the parameters. If we provide a function to the wand, we could find not only constant ( \pm a threshold $)$ data, but also predictable evolution, described by mathematical profiles. For instance, if we provide a function of the form $f(x)=T_{\text {hreshold }}+x$, by applying this function to the $\mathrm{X}$-axis, we can allow the user to automatically select curves with a single click.
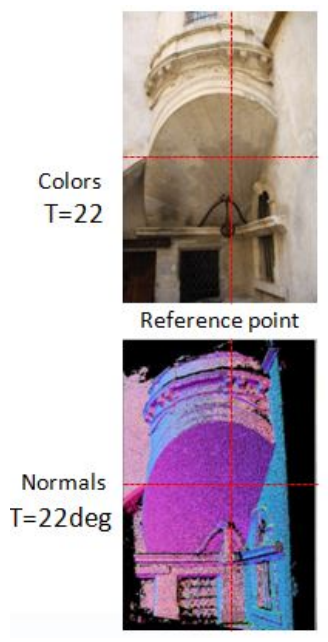

Fig. 6. Magic wand possibilities on normal map and colored textures

These tools offer the user a range of interactions according to his description objectives. These different tools are flexible enough to allow the ergonomic creation of regions for less experienced users, but also to offer precise annotation support to users eager to make the most of the tool's capabilities. These tools are designed to support and facilitate the user's annotation work in an interactive way.

These two methods of automatic region extraction are not disjointed; we can indeed pre-establish annotations using a preliminary segmentation, and then complete and correct it manually or using graphical tools.

\section{CONCLUSION}

This paper describes an approach for the semantic annotation of heritage assets using a bijective relationship established by indexation, between a 3D representation of an object and all the associated oriented 2D images. Our objective is to establish a digital continuum linking the acquisition, interpretation and representation phases, by providing relevant annotation tools to help cultural heritage experts to enrich data collected on the study object. While this goal is reachable, a few questions still raise our attention.

\section{DISCUSSION AND FUTURE WORKS}

Our workflow must be simple and flexible, and must allow to compare the annotations made within collaborative and multidisciplinary frameworks. Our web architecture might render collaborative work on multiple devices easy, but it also raises the obvious problem of Internet access: without a connection (within archaeological sites for example), the application is not accessible, thus preventing in situ uses. Although the regular deployment of $4 \mathrm{G}$ antennas is gradually improving network coverage in remote areas, it is still worth considering alternatives. For instance, we are studying the feasibility of implementing an offline mode that allows users to pre-load their projects in order to perform their annotations locally. The data entered could then be updated at a later Internet connection. Unfortunately, this solution is not fully adapted for collaborative use, as it would require a postponedsynchronisation algorithm to allow multiple users to work simultaneously on the same offline project.

Furthermore, the core of Aioli is based on a multi-stereo correlation engine. Its limits are therefore intrinsically linked to the photogrammetric pipeline, both for calculation and visualization. These drawbacks in mind, users are required to take important precautions when acquiring raw data and respect the good practices related to this metrological method: choice of sensor, camera settings, covering, etc. On the other hand, any image or data compatible with the photogrammetry process is theoretically directly usable in Aioli. A long-term perspective would therefore be to work on these computation steps in order to extend our approach to different scientific imaging techniques (e.g. multispectral imagery).

Last but not least, the question of ergonomics and acceptability remains open: the research carried out so far demonstrates the possibility of creating new tools for the study of Cultural Heritage. However, a paradigm shift in this area will only occur if these tools are truly adapted to the needs and practices of experts, and sufficiently intuitive and flexible. The choice of semi-automatic methods for annotation was made for this reason: the results are certainly less direct, but this allows users to configure and correct the data obtained, whether by the segmentation parameters, or by refining the temporary regions made by magic wand and magnetic lasso tools. The Aioli collaborative cloud platform is already open for beta-testing, and all the tools detailed in this paper have been developed with the aim of being integrated soon. Following the various expert feedback, further developments are focused on modalities for visualization and data capture, in order to facilitate observation and annotation of barely perceptible elements, and to establish a more tangible link between the digital clone and the real object. Indeed, despite the presence of ever more precise digital data, the study of built heritage still requires many round trips with the real object, to contextualize the data and compare it with what can be perceived in situ. From this perspective, Augmented and Mixed Reality appears to be a high-potential medium, on which we are currently working, but which requires requires solving the problem of the alignment of a project's data with the real heritage object. 


\section{REFERENCES}

[1] M. Doerr, "Ontologies for cultural heritage," in Handbook on Ontologies. Springer Berlin Heidelberg, 2009, pp. 463-486.

[2] T. Messaoudi, P. Véron, G. Halin, and L. De Luca, "An ontological model for the reality-based $3 \mathrm{~d}$ annotation of heritage building conservation state," Journal of Cultural Heritage, vol. 29, pp. 100-112, jan 2018. [Online]. Available: https://doi.org/10.1016/j.culher.2017.05.017

[3] K. Petridis, D. Anastasopoulos, C. Saathoff, N. Timmermann, Y. Kompatsiaris, and S. Staab, "M-OntoMat-annotizer: Image annotation linking ontologies and multimedia low-level features," pp. 633-640, 2006.

[4] S. Wefers, A. Karmacharya, and F. Boochs, "Development of a platform recommending 3d and spectral digitisation strategies," Virtual Archaeology Review, vol. 7, no. 15, p. 18, nov 2016. [Online]. Available: https://doi.org/10.4995/var.2016.5861

[5] S. Havemann, V. Settgast, R. Berndt, O. Eide, and D. W. Fellner, "The Arrigo Showcase Reloaded - towards a sustainable link between 3D and semantics," in VAST: International Symposium on Virtual Reality, Archaeology and Intelligent Cultural Heritage, M. Ashley, S. Hermon, A. Proenca, and K. Rodriguez-Echavarria, Eds. The Eurographics Association, 2008.

[6] F. Robbiano, M. Attene, M. Spagnuolo, and B. Falcidieno, "Partbased annotation of virtual 3d shapes," in Proceedings of the 2007 International Conference on Cyberworlds, ser. CW '07. Washington, DC, USA: IEEE Computer Society, 2007, pp. 427-436. [Online]. Available: https://doi.org/10.1109/CW.2007.53

[7] F. I. Apollonio, V. Basilissi, M. Callieri, M. Dellepiane, M. Gaiani, F. Ponchio, F. Rizzo, A. R. Rubino, R. Scopigno, and G. Sobra', "A 3d-centered information system for the documentation of a complex restoration intervention," Journal of Cultural Heritage, vol. 29, pp. 89 - 99, 2018. [Online]. Available: http://www.sciencedirect.com/science/article/pii/S1296207417301905

[8] F. Soler, F. J. Melero, and M. V. Luzón, "A complete 3d information system for cultural heritage documentation," Journal of Cultural Heritage, vol. 23, pp. 49 - 57, 2017. [Online]. Available: http://www.sciencedirect.com/science/article/pii/S1296207416302576

[9] W. Shi, E. Kotoula, K. Akoglu, Y. Yang, and H. Rushmeier, "CHEROb: A Tool for Shared Analysis in Cultural Heritage," in Eurographics Workshop on Graphics and Cultural Heritage, C. E. Catalano and L. D. Luca, Eds. The Eurographics Association, 2016.

[10] M. Potenziani, M. Callieri, M. Dellepiane, M. Corsini, F. Ponchio, and R. Scopigno, "3dhop: 3d heritage online presenter," Computers \& Graphics, vol. 52, pp. 129 - 141, 2015. [Online]. Available: http://www.sciencedirect.com/science/article/pii/S0097849315001041

[11] Sketchfab, "Sketchfab : 3d content on web, mobile, ar \& vr," 2012. [Online]. Available: http://sketchfab.com

[12] Schuetz, "Potree: Rendering large point clouds in web browser," 2016 [Online]. Available: http://potree.org

[13] N. Graf, "smapshot - geolocate our images on a virtual 3d globe!” 2017.

[14] P. Brivio, L. Benedetti, M. Tarini, F. Ponchio, P. Cignoni, and R. Scopigno, "Photocloud: Interactive remote exploration of joint $2 \mathrm{~d}$ and 3d datasets," IEEE Computer Graphics and Applications, vol. 33, no. 2, pp. 86-96, c3, March 2013.

[15] N. Snavely, S. M. Seitz, and R. Szeliski, "Photo tourism: Exploring photo collections in 3d," ACM Trans. Graph., vol. 25, no. 3, pp. 835-846, Jul. 2006. [Online]. Available: http://doi.acm.org/10.1145/1141911.1141964

[16] L. De Luca, C. Busayarat, C. Stefani, P. Véron, and M. Florenzano, "Semantic 3d media and content: A semantic-based platform for the digital analysis of architectural heritage," Comput. Graph., vol. 35, no. 2, pp. 227-241, Apr. 2011. [Online]. Available: http://dx.doi.org/10.1016/j.cag.2010.11.009

[17] C. Stefani, X. Brunetaud, S. Janvier-Badosa, K. Beck, L. De Luca, and M. Al-Mukhtar, "Developing a toolkit for mapping and displaying stone alteration on a web-based documentation platform," Journal of Cultural Heritage, vol. 15, no. 1, pp. 1-9, jan 2014. [Online]. Available: https://doi.org/10.1016/j.culher.2013.01.011

[18] Aioli, "A reality-based 3d annotation cloud platform for the collaborative documentation of cultural heritage artefacts," 2018. [Online]. Available: http://aioli.cloud

[19] M. Pierrot-Deseilligny, L. De Luca, and F. Remondino, "Automated image-based procedures for accurate artifacts $3 \mathrm{~d}$ modeling and orthoimage generation," Geoinformatics FCE CTU, vol. 6, no. 0, pp. 291-299, dec 2011. [Online]. Available: https://doi.org/10.14311/gi.6.36

[20] A. Manuel, P. Véron, and L. De Luca, "2D/3D Semantic Annotation of Spatialized Images for the Documentation and Analysis of Cultural Heritage," in Eurographics Workshop on Graphics and Cultural Heritage. The Eurographics Association, 2016.

[21] F. Duguet, G. Drettakis, D. Girardeau-Montaut, J.-L. Martinez, and F. Schmitt, "A Point-Based Approach for Capture, Display and Illustration of Very Complex Archeological Artefacts," in International Symposium on Virtual Reality, Archeology and Cultural Heritage (VAST2004). Bruxelles, Belgium: Eurographics, Dec. 2004, pp. 105-114.

[22] E. Grilli, F. Menna, and F. Remondino, "A review of point clouds segmentation and classification algorithms," ISPRS : The International Archives of the Photogrammetry, Remote Sensing and Spatial Information Sciences, vol. XLII-2/W3, pp. 339-344, 2017.

[23] M. Wang and Y.-H. Tseng, "Lidar data segmentation and classification based on octree structure," International Society of Photogrammetry and Remote Sensing and Spatial Information Sciences, pp. 12-23, 2004.

[24] A.-V. Vo, L. Truong-Hong, D. F. Laefer, and M. Bertolotto, "Octreebased region growing for point cloud segmentation," ISPRS Journal of Photogrammetry and Remote Sensing, vol. 104, pp. 88 - 100, 2015.

[25] Docker, "Docker : Build, ship and run apps anywhere," 2013. [Online]. Available: http://docker.com

[26] S. R. Vantaram and E. Saber, "Survey of contemporary trends in color image segmentation," Journal of Electronic Imaging, vol. 21, no. 4, p. 040901-1, oct 2012. [Online]. Available: https://doi.org/10.1117/1.jei.21.4.040901

[27] A. Ioannidou, E. Chatzilari, S. Nikolopoulos, and I. Kompatsiaris, "Deep learning advances in computer vision with 3d data: A survey," ACM Comput. Surv., vol. 50, no. 2, pp. 20:1-20:38, Apr. 2017. [Online]. Available: http://doi.acm.org/10.1145/3042064 